\title{
A Theory of Misperception in a Stochastic Dominance Framework and its Application to Structured Financial Products
}

April 21, 2016

\begin{abstract}
In this paper, the mechanism of misperception leading retail investors to investment choices, which are not the most profitable, is studied in a stochastic dominance framework from a theoretical perspective and supported by an extensive numerical analysis.

The theoretical contribution of the paper consists in the introduction of a specific definition of stochastic dominance, to capture the effects of an asymmetric trend-type misperception. Such a novel conceptualization is consistent with the perspective here adopted, i.e. the misperception is driven by a positive trend affecting the entire set of possible realizations.

The financial relevance of the theoretical proposal is highlighted through the paradigmatic case of structured financial products. To this end, we perform a pairwise numerical comparison between investment products to get insights about the inversion of the order of stochastic dominance, leading investors to prefer the less profitable instrument. The critical trend, the value where preferences are reversed, is interpreted as a measure of investors' misperception and compared with different levels of the volatility.

Some behavioral finance-type arguments provide insights on the interpretation of the obtained results.
\end{abstract}

Keywords: Behavioral finance, stochastic dominance, Monte Carlo simulations, framing effect, structured financial products, volatility.

JEL Classification: C65; D81; G24.

\section{Introduction}

Rationality is certainly not the unique route leading to financial decision-making. Psychology and human judgments must also be taken into account since random outcomes are not always objectively evaluated, and their realizations can be reinterpreted and misperceived by decision-makers. Often, 
these subjective biases lead to objectively not profitable financial choices.

In this paper, we provide a theoretical analysis of the stylized mechanism of the misperception that can explain the preferences granted to certain financial products. In a pairwise comparison, such a device allows analyzing cases of distortions of the risk-return profile, leading to the choice of the worst product.

By a theoretical perspective, we introduce a decision criterion - based on stochastic dominance - to analyze the mechanism of misperception. To this end, we appropriately adapt the classical definition of stochastic dominance to include the misperception of random amounts. This is conceptualized through the introduction of a positive trend, affecting the entire set of possible realizations of the worst financial product.

We conjecture that the roots of the popularity of some financial products among retail investors can be found in the poor understanding of these investments, driven not only by financial illiteracy but also by psychological factors. ${ }^{1}$. Indeed, such a popularity appears to be difficult to explain with investments profitability. In this respect, the perceived trend can be viewed as a psychological bias determining a misperceived improvement of the performances of the worst product. The conjecture we address finds its foundations in the main insights of behavioral finance. In agreement with some previous relevant contributions in financial economics, a volatility issue is also tackled using numerical analysis. We move from the fact that as the volatility changes, the magnitude of misperception changes as well.

To sum up, the novelties addressed by this paper are as follows.

From a theoretical point of view, we introduce a new definition of stochastic dominance. Such a new conceptualization is based on the assumption that misperception can be driven by a distortion of global trend-type. By a financial perspective, the proposed approach allows showing that, when purchasing, the retail investor must be put in the position of uniquely understanding the risk-return characteristics of a financial product. Indeed, financial illiteracy and/or ambiguous framing of a financial product can lead retail investors to misperception of global trend-type and, consequently, to not profitable (in terms of stochastic dominance) decisions.

The stylized mechanism of misperception is numerically implemented to analyze the paradigmatic case of two particular structured financial products. Indeed, some evidence suggests that especially among retail investors, some types of complex structured products are more popular than simple ones, although the latter should be preferred. Furthermore, via extensive simulations, we study how the volatility might influence the retail investor's choice. In so doing, relevant insights are gained on the relationship between the magnitude of misperception and riskiness of the financial product.

\footnotetext{
${ }^{1}$ There is no room here to focus on the source of such a poor understanding. We just point out that some evidence on the responsibility of financial institutions in exploiting investors' psychological biases is well documented. More details are provided in the last section.
} 
The importance of such a relationship is confirmed by several remarkable contributions in this field of research.

Theoretical and numerical results are interpreted in the light of the principles of behavioral finance, with a a particular reference to framing effect.

The remaining part of the paper is organized as follows: next section contains a brief review of the reference literature; Section 3 presents the theoretical foundation of the study; Section 4 provides details on numerical analysis; in Section 5 we discuss the findings on the relationship between misperception and volatility, and some conclusive remarks are provided.

\section{Literature review}

A proper contextualization of this paper can be identified in the broad field of the theory of financial decision making. From a theoretical perspective, particular attention is paid to the criteria for selecting a financial asset from a set of available alternatives. As selection criteria, we choose stochastic dominance because of its generality. Indeed, stochastic dominance is based on the distribution functions of random outcomes and allows to take into account all the characteristics of random variables (i.e.: fat tails, kurtosis, asymmetry, etc.). This decision criterion has been developed in Fishburn (1964); Hadar and Russel (1969); Hanoch and Levy (1969); Rothschild and Stiglitz (1970); Scarsini (1986) and, more recently, Kuosmanen (2004) and Osuna (2013). A survey on stochastic dominance can be found in Levy (2006) and in the recent monograph by Sriboonchitta et al. (2010), where several applications are also presented. In addition, it should be stressed that, as the recent paper by Muller et al.'s (2015) highlights, the theoretical advancements of stochastic dominance are still at the center of the scientific debate.

Here, we differ from the quoted contributions, because we aim to build a decision criterion which is specifically tailored on a misperception of global trend type ${ }^{2}$. We introduce a distortion in the random amounts and then apply stochastic dominance to distorted quantities, without modifying the original definition of the decision criterion. This approach provides a very general rule for analyzing the mechanism of misperception and extends the paper by Castellano and Cerqueti (2013), in which the restrictive cases of constant trend and lump sum were considered. Such an extension also allows a deep analysis of the riskiness and its connections with the biases which often characterize the evaluation of random amounts.

Another area of the literature to which this paper refers to is the general psychological analysis of decision processes. Some empirical studies show how the presence of psycho-biases can affect the selection process from a set of available alternatives. In this respect, several paradoxes arise. In

\footnotetext{
${ }^{2}$ The definition of stochastic dominance we provide does not aim to describe local convexities of the utility functions or other properties of the preference rules.
} 
general, behavioral finance scientists have provided a systematization of the psycho biases and their impact on the financial decisions (see Fellner et al., 2009, and references therein). One of the most noticeable ones is that individuals tend to revert their preferences when even identical alternatives are presented at different times (see e.g. Camerer, 1989, Starmer and Sugden, 1989, Wu, 1994, Hey and Orme, 1994). Cross et al. (2005) discuss the presence of tensions driving the mechanism of mispricing in the particular case of the minimal personality of investors. Lamba and Seaman (2008) deal with a pricing model with heterogeneous agents by introducing into the evaluation scheme also the sentiment. Such a variable is driven by several psychological factors and can be written as the average of the states $(+1$ as the long position and -1 as short position) of all investors. When a decision involves the evaluation of good and bad performances, irrationality can also be motivated by the so-called disposition effect, i.e. the tendency showed by investors to sell the assets experiencing a positive trend in the price and keep the ones whose price has decreased. The disposition effect explains the occurrence of a different perception between bad and good realizations in investors' mind. In this respect, Friedman and Savage (1948) and Markowitz (1952) theorize the existence of switches in investors' mind between risk-aversion and risk-seeking, in correspondence of some critical outcomes.

Ritter (2003) highlights some specific causes of investors' irrationality. Among them, one of the most popular is the so-called framing effect which refers to an ambiguous form of presentation of available alternatives, so that a distorted framing of alternative proposals can lead to the reversal of preferences. Kahneman and Tversky (1979) conduct experimental psychological researches and provide an empirical validation of Friedman, Savage, and Markowitz's speculations, formalizing one of the founding principles of prospect theory. In line with prospect theory, Rieger (2011) applies a behavioral finance argument to deal with financial decisions and design of structured financial products. The framing effect is acknowledged to be responsible for a significant number of misperception mechanisms (see e.g. Berger and Smith, 1998, Wang and Fishbeck, 2004, Breuer and Prest, 2007, Bernard et al., 2011, Rieger, 2012).

These quoted papers are very close to our point of view. In particular, Bernard et al. (2011) provide a valuable reference for the selection mechanism from a set of structured financial products. They show that investors have a tendency to overprice the most complex ones and argue that such a misreading is driven by the optimism with which financial institutions frame the prospectuses of investments to retail investors. This paper differs from that of Bernard et al. (2011) in many respects. The most important one is that we abandon the classical mean-variance approach and develop the analysis in the broader framework of stochastic dominance, providing a methodological advancement in decision theory.

For the sake of completeness, we remind the reader that is possible to describe the individuals' subjectivity, violations of reasonable choices and related implications also by adopting a possibilistic 
approach (Inuiguchi and Ramik, 2000, Popescu and Fulga, 2011). Such a way to proceed is associated with concepts like ambiguity and vagueness which request the use of fuzzy theory. In any case, such a perspective is very far from the stochastic approach adopted in this paper.

\section{The theoretical framework}

In this section, the theoretical contribution of the paper is presented. In particular, the focus is on the violation of stochastic dominance induced by a mechanism of misperception.

Consider two random variables $X$ and $Y$, with distribution functions $F_{X}$ and $F_{Y}$ respectively. The following assumption holds true throughout the paper:

Assumption 1. The random variables $X$ and $Y$ have bounded support.

We also assume that $X$ and $Y$ represent two stochastic outcomes, and rational investors take a financial position after evaluating $X$ and $Y$. To this end, investors use stochastic dominance criterion as a decision rule. More specifically, for a given $n \in \mathbb{N}$, if $X$ stochastically dominates $Y$ of order $n$, i.e.: $X>_{n} Y$, then the decision maker should prefer $X$ to $Y$. Tthis does not always occur. The fact that some investors assess $Y$ better than $X$ may depend on the misreading of the realizations of $X$, or $Y$, or both. As already discussed in Castellano and Cerqueti (2013), the misperception can be due to an optimistic view of the worst product, induced by a constant trend. Here, we extend this result and assume that the stylized rule describing the misperception of the worst random outcome is caused by a general deterministic trend, not necessarily constant, that favorably affects the realizations of $Y$.

An optimistic trend can be defined using a bounded function $\alpha: \mathbb{R} \rightarrow[0,+\infty)$. The perturbation of $Y$ induced by the trend $\alpha$ generates a new random variable $Y_{\alpha}$. The formal definition of $Y_{\alpha}$ can be easily derived from $Y$, by translating the cumulative distribution function $F_{Y}$ :

$$
F_{Y_{\alpha}}(r)=F_{Y}(r-\alpha(r)), \quad \forall r \in \mathbb{R}
$$

It is worth noting that if $\alpha$ is bounded in $\mathbb{R}$, then $F_{Y_{\alpha}}$ is a cumulative distribution function. Obviously, if $\alpha \equiv 0$ no misperception occurs.

Assumption 2. The decision makers have complete information on $F_{X}$ and $F_{Y_{\alpha}}$.

In particular, Assumption 2 states that investors evaluate the random outcome $X$ correctly and make an incorrect assessment of the outcome $Y$. The latter is induced by a psycho-distortion which takes the form of a deterministic trend.

Remark 1. No mathematical assumptions on the behavior of $\alpha$ with respect to its argument are required to introduce the concept of optimistic trend. This let the definition of trend be rather general. However, a nondecreasing a formalizes the specific cases in which the larger the amount to be distorted, the bigger the size of the distortion. 
A specific definition of stochastic dominance is introduced to capture the effect of a trend-type misperception.

Definition 1. Consider $n \in \mathbb{N}$, two random outcomes $X$ and $Y$ and a bounded optimistic trend $\alpha: \mathbb{R} \rightarrow[0,+\infty)$.

The random outcome $Y \alpha$-dominates of order $n X\left(Y>_{\alpha, n} X\right.$, hereafter $)$ if and only if $Y_{\alpha}$ stochastically dominates $X$ of order $n \in \mathbb{N}\left(Y_{\alpha}>_{n} X\right)$, i.e.:

$$
\left\{\begin{array}{l}
F_{Y_{\alpha}}^{(n)}(r) \leq F_{X}^{(n)}(r), \quad \forall r \in \mathbb{R} \\
\text { and } \\
\exists r^{*} \in \mathbb{R} \text { such that } F_{Y_{\alpha}}^{(n)}\left(r^{*}\right)<F_{X}^{(n)}\left(r^{*}\right),
\end{array}\right.
$$

where, for each $r \in \mathbb{R}$, the function $F_{X}^{(n)}$ is defined recursively as follows:

$$
F_{\bullet}^{(n)}(r)=\left\{\begin{array}{l}
F_{\bullet}(r), \quad \text { for } n=1 ; \\
\int_{-\infty}^{r} F_{\bullet}^{(n-1)}(s) \mathrm{d} s, \quad \text { for } n>1,
\end{array}\right.
$$

with $\bullet=X, Y_{\alpha}$.

The concept of $\alpha$-stochastic dominance can be seen as an asymmetric criterion for evaluating two random outcomes. Indeed, as the Definition 1 states, only one of the quantities involved in the comparison is distorted. Since the trend is assumed to be bounded, it is easy to check that $Y>_{\alpha, n} X$ and $X>_{\alpha, n} Y$ cannot hold simultaneously.

No closed form results for the reversal of stochastic dominance are available if the shape of the function $\alpha$ is not specified. Despite this, a sufficient condition can be easily and directly derived from the classical definition of stochastic dominance and Definition 1.

Proposition 1. Fix $n \in \mathbb{N}$ and assume that $X>_{n} Y$. Consider a bounded optimistic trend $\alpha: \mathbb{R} \rightarrow$ $[0,+\infty)$. There exists $\alpha_{n}^{\star}>0$ such that

$$
\lim _{r \rightarrow-\infty} \alpha(r) \geq \alpha_{n}^{\star} \quad \Rightarrow \quad Y>_{\alpha, n} X
$$

Proof. Assume that

$$
\alpha(r) \geq \alpha_{n}^{\star}, \quad \forall r \in \mathbb{R} .
$$

By (5) and by the definition of $F_{Y}$, we have that:

$$
F_{Y}^{(n)}(r-\alpha(r)) \leq F_{Y}^{(n)}\left(r-\alpha_{n}^{\star}\right), \quad \forall r \in \mathbb{R} .
$$

Now, first-order stochastic dominance implies stochastic dominance of order greater than 1 . So, by (6), we need to search for $\alpha_{1}^{\star}$ such that

$$
F_{Y}\left(r-\alpha_{1}^{\star}\right) \leq F_{X}(r), \quad \forall r \in \mathbb{R} .
$$


By Assumption 1, the quantities $X$ and $Y$ have bounded support. Therefore, there exist four scalars $r_{1}^{\star}, r_{2}^{\star}, r_{3}^{\star}, r_{4}^{\star} \in \mathbb{R}$ such that:

$$
\begin{cases}F_{X}(r)=0, & \text { for } r \leq r_{1}^{\star} ; \\ F_{X}(r) \in(0,1), & \text { for } r_{1}^{\star}<r<r_{2}^{\star} ; \\ F_{X}(r)=1, & \text { for } r \geq r_{2}^{\star},\end{cases}
$$

and

$$
\begin{cases}F_{Y}(r)=0, & \text { for } r \leq r_{3}^{\star} ; \\ F_{Y}(r) \in(0,1), & \text { for } r_{3}^{\star}<r<r_{4}^{\star} ; \\ F_{Y}(r)=1, & \text { for } r \geq r_{4}^{\star} .\end{cases}
$$

By the hypothesis that $X>_{n} Y$, we have that $r_{1}^{\star} \geq r_{3}^{\star}$ and $r_{2}^{\star} \geq r_{4}^{\star}$. A sufficient condition to invert the stochastic dominance relation between $X$ and $Y$ is obtained by translating the function $F_{Y}$ in order to have:

$$
F_{Y}\left(r-\alpha_{1}^{\star}\right)=0, \quad \text { for } r \leq r_{2}^{\star} .
$$

To get (10) it is sufficient to consider $\alpha_{1}^{\star}>r_{2}^{\star}-r_{3}^{\star}$.

The constant $\alpha_{n}^{\star}$ is the critical trend for stochastic dominance inversion, and can be defined as

$$
\alpha_{n}^{*}=\inf \left\{\alpha \in(0,+\infty) \mid Y>_{\alpha, n} X\right\}
$$

The subscript $n$ indicates that the critical threshold value of $\alpha$ depends on the order of stochastic dominance to be reversed.

A nice result, particularly suitable for real world applications, provides a link between constant and critical trend. With a reasonable abuse of notations, we denote the constant trend through a parameter $\alpha \in[0,+\infty)$, the misperception of $Y$ as $Y_{\alpha}$ and the cumulative distribution function of $Y_{\alpha}$ as $F_{Y_{\alpha}}$, so that (1) holds also with a constant trend, playing the role of critical trend introduced in Proposition 1 and formula (11).

The reversal of preference between $X$ and $Y$ is achieved only when $Y$ is misperceived through a large enough constant trend $\alpha$. This is formalized in the next Corollary of Proposition 1.

Corollary 1. Fix $n \in \mathbb{N}$ and assume that $X>_{n} Y$. Then $Y>_{\alpha, n} X$, for each $\alpha \geq \alpha_{n}^{\star}$, where $\alpha_{n}^{\star}$ is defined as in (11).

The following result explains the dependence between critical trends and corresponding orders of stochastic dominance.

Proposition 2. Consider $n_{1}, n_{2} \in \mathbb{N}$. It results $n_{1} \leq n_{2}$ if and only if $\alpha_{n_{1}}^{\star} \geq \alpha_{n_{2}}^{\star}$.

Proof. $n_{1} \leq n_{2}$, if and only if $Y>_{\alpha, n_{1}} X$ implies that $Y>_{\alpha, n_{2}} X$, for each $\alpha \in[0,+\infty$ ) (see Levy (2006), Chapter 3). By definition, we have $Y>_{\alpha_{n_{1}}^{\star}, n_{1}} X$. Hence, it results $Y>_{\alpha_{n_{1}}^{\star}, n_{2}} X$, and the definition of $\alpha_{n_{2}}^{\star}$ guarantees that $\alpha_{n_{1}}^{\star} \geq \alpha_{n_{2}}^{\star}$. 


\section{A numerical application}

In this section, we provide a numerical application of the theoretical proposal presented above. We refer to two stock market-linked products, falling into the category of structured investments. This is the name given to a broad range of products that, in the majority of cases, are issued by investment banks or insurance companies and sold to retail investors who appreciate the customization of a variety of instruments into one product and the frequent presence of a principal guarantee function which offers downside risk protection of the invested wealth, coupled with upside potential return in the stock market.

Structured products are very sophisticated tools and often retail investors are not able to clearly understand the financial risks associated with their complex structures. Besides, packaged retail investment products are the focus of a heated debate just as far as the rules for the presentation of their risk-return profile. Recently, Rieger (2012) finds that complexity might be a sophisticated method to exploit systematic biases in probability estimation of investors to make financial products look safer and more attractive than they are. Wallmeier (2011) states that the success of some of the most popular products is mainly due to behavioral biases of investors and advances concerns related to the complexity of these financial products, often accompanied by the little transparency of their prospectuses.

For these reasons, structured products are suitable to represent the object of the numerical application related to the stylized mechanism of misperception discussed above. At any rate, we like to emphasize here that our theoretical proposal provides general results, which are independent on the type of financial instruments considered here.

As in Castellano and Cerqueti (2013) and Bernard et al. (2011), we consider structured investments that, in the absence of default risk, provide a guaranteed minimum return, so that the final return is never less than a prespecified floor which is in place throughout the life of the contract. In addition, capped contracts are taken into account. This implies that, at maturity, the final payoff is given by a combination of periodic returns (locally capped contracts) or a bonus return (globally capped contracts), varying according to the performance of the underlying asset, between the issue and maturity date.

In appearance, although the two types of contract are very similar, they are characterized by different structures and properties. Indeed, the globally capped contract shows a simple financial structure that can be easily replicated and priced. Conversely, the locally capped contract is characterized by a more complex and not replicable structure which generates a path-dependent final payoff and, consequently, the difficulty of determining its fair price in closed form.

The final payoffs of globally floored-locally capped contracts and globally floored-globally capped 
are given respectively in (12) and (13) (see Boyle and Tse, 1990; Bernard et al., 2011):

$$
\begin{gathered}
Y_{T}=Y_{0}\left\{(1+F)+\max \left[0, \sum_{k=1}^{n T} \min \left(c, \frac{S_{t_{k}}}{S_{t_{k-1}}}-1\right)-F\right]\right\}, \\
X_{T}=X_{0}\left\{(1+F)+\max \left[0, \min \left(C, \frac{S_{T}}{S_{0}}-1\right)-F\right]\right\},
\end{gathered}
$$

with $T$ representing the maturity of the contract; $n$ the number of periods in a year; $Y_{0}$ and $X_{0}$ the amounts paid at the inception date, $t=0$, for each contract; $F$ the global floor (guaranteed minimum return at maturity); $c$ the local cap (maximum allowed periodical return); $C>F$ the global cap (maximum allowed return); $S_{t_{k}} \in[0, T]$ the prices of the underlying asset at prespecified dates $t_{k} ; S_{0}$ and $S_{T}$ the prices of the underlying at inception and maturity date, respectively.

Assuming that the price of the underlying asset follows a lognormal diffusion, these products are fairly priced in a risk neutral setting by combining Monte Carlo and optimization methods, according to the algorithm described in Castellano and Cerqueti (2013). Some ideas behind such algorithm are provided, to assist the reader.

Using a grid search procedure, the fair global and local caps, $C$ and $c$ respectively, are determined so as to make the price of the two contracts equal to an assigned value, $\widehat{P}$.

In particular, in a Black-Scholes-Merton's setting, we take the discounted expected payoff and solve for the global and local caps which minimize the distance of the risk neutral price from the given one, assuming all the other parameters as given.

We use the vector of parameters $\xi=\left[\begin{array}{llllllll}T & X_{0}=Y_{0} & F & r & \sigma & \delta & S_{0} & \widehat{P}\end{array}\right]$, for both simple and complex contracts which share the same underlying, $\left(S\right.$ with current price $\left.S_{0}=10\right)$, maturity $(T=5$ years), initial investment $\left(X_{0}=Y_{0}=1,000\right)$, guaranteed minimum return $(F=0.1)$, price $\widehat{P}=920$, discount factor $r=0.05$, volatility of underlying $\sigma=0.15$ and dividend yield $\delta=0.02 .^{3}$

The complicatted contract is based on a quarterly sum cap $(n=4)$ and its estimated fair cap is $\widehat{c}=8.67 \%$, while the estimated fair global cap level, is $\widehat{C}=30.53 \%$.

Since the products considered here depend on the assumed volatility of the underlying (classical references to this phenomenon are Avellaneda et al., 1995; Lyons, 1995; and Wilmott, 2000), we also provide an analysis based on volatility. This allows us to gain some insights on the relationship between the magnitude of misperception and riskiness of the underlying asset. Moreover, it is possible to compare the results of our numerical experiments with those obtained by Breuer and Perst (2007) who, investigating the demand for structured products, showed that the underestimation of volatility makes these products attractive to retail investors.

Starting with the vector of parameters:

$$
\xi^{\prime}=\left[\begin{array}{lllllllll}
T & X_{0}=Y_{0} & F & r & \widehat{c} & \delta & S_{0} & \widehat{C} & n
\end{array}\right],
$$

\footnotetext{
${ }^{3}$ The parameters selected for the application correspond to standard market assumptions. However, the results hold also for different vectors of parameters, $\xi$.
} 
we run our experiment for different levels of volatility in the underlying $(0 \% \leq \sigma \leq 100 \%$, with discretization step equal to $0.1 \%$ ), to plot the prices of the two contracts.

Using Monte Carlo, each data point is computed performing 10,000 replications. In Figure 1, the red line represents the present value, computed at time $t=0$, of the guaranteed redemption amount, calculated as $X_{0}(1+F) e^{-r T}=Y_{0}(1+F) e^{-r T}=856.68$.

\section{INSERT FIGURE 1 ABOUT HERE.}

Caption of Figure 1: Prices of globally and locally capped contracts as a function of volatility.

Looking at the plot reported in Figure 1, it can be noticed that prices of both contracts are not monotonic functions of the volatility, and the price of the complex product decreases faster than the one of the simple one, because of the truncation effects on large positive returns due to local caps.

\subsection{Comparison of random outcomes via stochastic dominance}

The next step of this application is related to the comparison of the random outcomes of the two considered contracts, via stochastic dominance criteria. To this end, we simulate the final payoffs for both contracts so that two distinct prospects, with cumulative distribution functions respectively given by $\widehat{F}_{X}$ and $\widehat{F}_{Y}$, are determined.

We recall that $X$ stochastically dominates $Y$ of order $n \in \mathbb{N}\left(X>_{n} Y\right)$ if and only if:

$$
\left\{\begin{array}{l}
\widehat{F}_{X}^{(n)}(r) \leq \widehat{F}_{Y}^{(n)}(r), \quad \forall r \in \mathbb{R} \\
\text { and } \\
\exists r^{*} \in \mathbb{R} \text { such that } \widehat{F}_{X}^{(n)}\left(r^{*}\right)<\widehat{F}_{Y}^{(n)}\left(r^{*}\right) .
\end{array}\right.
$$

To verify (14) and check whether $X$ dominates $Y$ of order $n$, we simulate $j=1, \ldots, K(K=10,000)$ prospects, named $X^{(j)}$ and $Y^{(j)}$, and compute:

$$
\Psi_{K}^{(n)}=\frac{1}{K} \sum_{j=1}^{K} 1\left(\widehat{D}_{j}^{(n)}(r) \leq 0, \forall r \in \mathbb{R} \text { and } \exists r^{*}: \widehat{D}_{j}^{(n)}\left(r^{*}\right)<0\right)
$$

where:

$$
\widehat{D}_{j}^{(n)}(r)=\widehat{F}_{X^{(j)}}^{(n)}(r)-\widehat{F}_{Y^{(j)}}^{(n)}(r)
$$

and $1(\cdot)$ denotes the indicator function. Then the Law of Large Numbers in strong form is applied:

$$
\Gamma_{j}= \begin{cases}1, & \text { if } \widehat{D}_{j}^{(n)}(r) \leq 0, \forall r \in \mathbb{R} \text { and } \exists r^{*}: \widehat{D}_{j}^{(n)}\left(r^{*}\right)<0 \\ 0, & \text { otherwise. }\end{cases}
$$

to derive, with probability one, the average number of times in which $X$ stochastically dominates $Y$. 
This procedure is applied for different values of the volatility of the underlying, $\sigma$ (varying between $0 \%$ and $70 \%$, with a discretization step equal to $0.1 \%$ ). The results referring to first, second and third order stochastic dominance are summarized in Table 1, which reports (in mean) the percentages of cases in which the simple contract dominates the complex one. In the first column, the ranges of the volatility of the underlying are reported. The second column presents the percentage of first-order stochastic dominance $X>_{1} Y$ occurrences, while third and fourth columns report second and thirdorder stochastic dominance occurrences, respectively. Results are obtained running the procedure to obtain 10,000 prospects, for each contract and volatility level.

\section{INSERT TABLE 1 ABOUT HERE.}

Caption of Table 1: The relation between volatility ranges and percentage (in mean) of first, second and third stochastic dominance occurrences.

The comparison of the two distributions for different volatility ranges partially contradicts what occurs on the retail market and highlights the problem of not profitable (in a stochastic dominance framework) investors' choices. In particular, even though evidence suggests that the complex contract, $Y$, is more popular than the simple one, $X$, we find that - according to stochastic dominance criteria - investors should prefer the simple contract, $X$. More details on this are discussed in the next section.

Another step of the numerical application is related to the mechanism of misperception, which describes the process that leads retail investors to select complex products in place of simple ones. Starting from the theoretical results, we perturb the cumulative distribution function of the contract $Y$, to map $Y$ into $Y_{\alpha}$, in the case of a critical trend $\alpha^{*}$ (see Proposition 1), in the attempt of providing a measure of the magnitude of misperception. First and second-order stochastic dominance criteria are taken into account. Hence, for each level of volatility, we proceed as follows.

- Given the $j=1, \ldots, K$ prospects, select an integer $v$ and a $v$-dimensional positive vector, $\Delta=\left(\Delta^{(l)}\right)_{l=1, \ldots, v}$, whose elements are the values to be assigned to the unknown constant trend, $\alpha$.

- Perturb $Y$ through $\Delta$ and set the vector $Y_{\alpha}=\left(Y_{\alpha}^{(l)}\right)_{l=1, \ldots, v}$.

- Obtain $X^{(l)}$, with $l=1, \ldots, v$.

- Evaluate, for each element $l^{t h}$ of the $v$-dimensional vector $\Delta$, the stochastic dominance criteria as determined by the perturbation of the complex contract, $Y$, as above. In particular, in order to verify the $\alpha$-stochastic dominance, we compute:

$$
\Phi_{v}^{(n)}=\frac{1}{v} \sum_{l=1}^{v} 1\left(\widehat{\alpha}_{l}^{(n)}(r) \leq 0, \forall r \in \mathbb{R} \text { and } \exists r^{*}: \widehat{\alpha}_{\alpha}^{(n)}\left(r^{*}\right)<0\right)
$$


where:

$$
\widehat{\alpha}_{\alpha}^{(n)}(r)=\widehat{F}_{Y_{\alpha}^{(l)}}^{(n)}(r)-\widehat{F}_{X^{(l)}}^{(n)}(r) .
$$

- Find the minimum value $l^{*} \in\{1, \ldots, v\}$ such that $Y_{\alpha}^{\left(l^{*}\right)}>_{n} X^{\left(l^{*}\right)}$.

\section{INSERT TABLE 2 ABOUT HERE.}

Caption of Table 2: The relation between volatility ranges and magnitude in misperception, for first and second order stochastic dominance occurrences.

Table 2 summarizes all the results related with the magnitude of misperception for different ranges of volatility, by reporting the values of critical thresholds, $\alpha_{n}^{\star}$ 's. Panel A shows the percentages of occurrence of $Y>_{\alpha_{1}^{\star}, 1} X$, for different levels of $\alpha_{1}^{\star}$, while panel B contains the percentages of occurrence of $Y>_{\alpha_{2}^{\star}, 2} X$, for different levels of $\alpha_{2}^{\star}$.

\section{Analysis of the results and concluding remarks}

The first-order stochastic dominance, which is related to investors characterized by strictly increasing utility function (see Levy 2006, Theorem 3.1), does not allow to draw any conclusion, since there is no evidence of dominance, for all levels of volatility (see Table 1).

On the contrary, results related to second-order stochastic dominance point out that investors characterized by risk-adverse utility functions (Levy, 2006, Theorem 3.2) and being expected utility maximizer should prefer the simple contract $X$. This finding is robust in the case of very low and high volatility ranges. For instance, it can be observed that for $\sigma<0.033$ and $\sigma>0.164$, we have that $X>_{2} Y$ in $100 \%$ of cases. On the contrary, for $\sigma \in(0.033,0.164)$ we cannot provide straightforward results. However, it is possible to show that, ceteris paribus, locally capped have higher fair prices than the globally capped contracts, for a small range of volatility (Figure 2). This outcome supports the argument related with the preference that retail investors should accord - after an objective evaluation of the products - to simple contracts rather than complex ones.

\section{INSERT FIGURE 2 ABOUT HERE.}

Caption of Figure 2: The distance between prices of global and local cap contracts as a function of volatility.

The numerical experiments based on third-order stochastic dominance provide significant results for the simple contract since, for each level of volatility, we have that $X>_{3} Y$ in $100 \%$ of cases. It is worth to recall here that the third-order stochastic dominance implies the attractive feature of 
decreasing absolute risk aversion (a non-negative third derivative of the utility function, see Levy, 2006, Theorem 3.3). This finding ensures that $Y$ cannot stochastically dominate $X$ for any order. Indeed, the stochastic dominance of a given order $n$ implies the dominance also for orders greater than $n$, but $X>_{3} Y$ excludes that $Y$ can be preferred to $X$ under first and second-order stochastic dominance.

In light of these results, the popularity of locally capped contracts on globally capped ones seems to be counterintuitive, since there is no reason to prefer the complex product to the simple one, at least in a pairwise comparison.

The popularity of complex products can be explained by the mechanism of misperception. In this respect, Table 2 shows what happens to the relation between simple and complex contract, when a distorting trend is in operation. We stress that the magnitude of the misperception is inversely related to the level of $\alpha^{*}$ since, when the stochastic dominance is violated for low levels of $\alpha^{*}$, investors are more likely to perceive a biased risk-return profile of the investment.

When the volatility is low, the violation of first-order stochastic dominance occurs for a small threshold, $\alpha_{1}^{\star}$. For $\alpha_{1}^{\star}=0.03$ and $\sigma<0.096$, the locally capped $\alpha$-dominates globally capped contract in the $95 \%$ of cases (on average). The higher the volatility level, the greater the value of $\alpha_{1}^{\star}$ which is responsible of the inversion in the first-order stochastic dominance for all the cases. Specifically, moving to $\alpha_{1}^{\star}=0.18$, the complex product $\alpha$-dominates the simple one in the entire set of cases, regardless of the volatility level.

Regarding the second-order stochastic dominance, results show that $Y>_{\alpha, 2} X$ in $100 \%$ of cases for $\alpha_{2}^{\star}=0.03$ and $\sigma<0.135$. No evidence of stochastic dominance occurs when $\sigma>0.2$. Moreover, $Y>_{\alpha, 2} X$ in $100 \%$ of cases when $\alpha_{2}^{\star} \geq 0.06$, regardless of volatility levels.

The latter results help to explain why investors prefer complex products. The mechanism of misperception drives to not optimal decisions because the distortion in the entire set of realizations of the complex product reverses the direction of the stochastic dominance. A result of particular interest is provided by the analysis of the magnitude of misperception as a function of volatility. Evidence are very clear in confirming that the magnitude of misperception is higher for low volatility levels. This is consistent with the empirical findings discussed in several other financial studies. Breuer and Prest (2007) showed that investors who underestimate the volatility of the underlying are particularly interested in structured products. Other classical references are Modest and Sundaresan (1983), Figlewski (1984), MacKinlay and Ramaswamy (1988) and Yadav and Pope (1994). All these papers stress that the magnitude of distortions is greater in cases of low volatility.

Moving to the interpretation of the obtained results, some remarks on the motivations which might lead to misperception are necessary.

We proceed from the fact that, in the peculiar case of structured products, the behavior of retail investors is driven by the confidence towards the financial institution which supplies investment 
products. In this respect, financial institutions are in the position of exploiting the naivety of households by presenting some financial products in a too optimistic or ambiguous fashion, overweighting their extremely positive performances. This might push investors to misperception and, hence, not profitable (in a stochastic dominance framework) decisions. In this respect, we refer the interested reader to Bernard et al. (2011) where real cases of optimistic prospectuses proposed by a financial institution on the future performances of globally floored-locally capped contracts are presented. Other references in support of the conjecture are cited below

Wallmaier (2011), highlights that usually the information provided to investors is focused on payoff diagrams ad suggests that, for the transparent development of the structured product market, it is important to improve investors' information and understanding. To this end, he analyzes the information requirements to debias investors and proposes a risk-return survey to provide relevant and comprehensive information on market risk. Rieger (2012), presents results of an experimental study focusing on probability estimates, in the context of particular classes of structured products, and concludes that to debias investors it would be optimal to provide historical probabilities for both the probability to touch a barrier and the probability to end below the barrier, at maturity. Henderson and Pearson (2011), for some structured products, find that investors would likely have been better off investing in non-interest bearing accounts. They state that this result is consistent with the idea that financial institutions hide some aspects of the terms on which their products are offered. Hens and Rieger $(2009,2012)$ show that the most popular products derive their success not from rational choices, but from behavioral factors like framing, loss aversion and misestimation of probabilities. In particular, the current popularity of some structured products can be explained with clarity taking into account only the misestimation of probabilities. Thus, they come to the conclusion that the market of structured products represents a huge business for banks and suggest to improve the understanding of these financial instruments by customers through the presentation of probabilistic scenarios ${ }^{4}$.

\section{References}

[1] Avellaneda M., Levy A., Paras A. (1995). Pricing and hedging derivative securities in markets with uncertain volatilities. Applied Mathematical Finance 2, 73-88.

\footnotetext{
${ }^{4}$ It is worth citing the case of the Lawyers Burke and Stoltmann that, in Illinois, presented a class-action to recover losses against Wall Street brokerage firms. In their investigation, Burke and Stoltmann highlight that structured products were favorably presented to investors and inappropriately depicted as $100 \%$ safe and secure.

More information on http://www.structuredproductfraud.com/ and http://risktransparency.altervista.org/.
} 
[2] Berger, P.D., Smith, G.E. (1998). The Impact of Prospect Theory Based Framing Tactics on Advertising Effectiveness. Omega, International Journal of Management Science 26(5), 593-609.

[3] Bernard C., Boyle P.P., Gornall W. (2011). Locally-Capped Investment Products and the Retail Investor. Journal of Derivatives 18(4), 72-88.

[4] Boyle P.P., Tse Y.K. (1990). An algorithm for computing values of options on the maximum or minimum of several assets. Journal of Financial and Quantitative Analysis 25, 215-227.

[5] Boyle P.P., Turnbull S.M. (1989). Pricing and Hedging Capped Options. The Journal of Futures Markets 9, 41-54.

[6] Breuer W., Perst A. (2007). Retail banking and behavioral financial engineering: the case of structured products. Journal of Banking and Finance 31, 827-844.

[7] Camerer, C.F. (1989). An Experimental Test of Several Generalized Utility Theories. Journal of Risk and Uncertainty 2, 61-104.

[8] Castellano R., Cerqueti R. (2013). Roots and effects of financial misperception in a stochastic dominance framework. Quality and Quantity, 43, 3371-3389.

[9] Cross R., Grinfeld M., Lamba H., Seaman T. (2005). A threshold model of investor psychology. Physica A 354, 463-478.

[10] Fellner G., Güth W., Maciejovsky, B. (2009). Satisficing in financial decision making: a theoretical and experimental approach to bounded rationality. Journal of Mathematical Psychology $53,26-33$.

[11] Figlewski S. (1984). Hedging Performance and Basis Risk in Stock Index Futures. Journal of Finance 39, 657-670.

[12] Fishburn P.C. (1964). Decision and Value Theory. Wiley, New York.

[13] Friedman M., Savage L.J. (1948). The utility analysis of choices involving risk. Journal of Political Economy 56, 279-304.

[14] Hadar J., Russell W.R. (1969). Rules for ordering uncertain prospects. American Economic Review 59, 25-34.

[15] Hanoch G., Levy H. (1969). The efficiency analysis of choices involving risk. Review of Economic Studies 36, 335-346.

[16] Henderson, B.J., Pearson, N.D. (2007). Patterns in the Payoffs of Structured Equity Derivatives. AFA 2008 New Orleans Meetings Paper. Available at SSRN: http://ssrn.com/abstract=972570 or http://dx.doi.org/10.2139/ssrn.972570 
[17] Hens T., Rieger M.O. (2009). The dark side of the moon: structured products from the customer's perspective, EFA 2009 Bergen Meetings.

[18] Hens T., Rieger M.O. (2012).Explaining the demand for structured financial products: survey and field experiment evidence. Z Betriebswirtsch 82, 491-508.

[19] Hey, J.D., Orme, C. (1994). Investigating Generalisations of Expected Utility Theory Using Experimental Data. Econometrica 62, 1291-1326.

[20] Inuiguchi, M., Ramik, J. (2000). Possibilistic linear programming: a brief review of fuzzy mathematical programming and a comparison with stochastic programming in portfolio selection problem. Fuzzy Sets and Systems, 111(1), 3-28.

[21] Kahneman D., Tversky A. (1979). Prospect theory: an analysis of decision under risk. Econometrica 47, 263-291.

[22] Kuosmanen, T. (2004). Efficient diversification according to stochastic dominance criteria. Management Science, 50, 1390-1406.

[23] Lamba H., Seaman T. (2008). Rational expectations, psychology and inductive learning via moving thresholds. Physica A 387, 3904-3909.

[24] Levy H. (2006). Stochastic Dominance: Investment Decision Making Under Uncertanity. Kluwer Academic Publishers, Boston MA.

[25] Lyons T.J. (1995). Uncertain volatility and the risk-free synthesis of derivatives, Applied Mathematical Finance 2, 117-133.

[26] MacKinlay C.A., Ramaswamy K. (1988). Index Futures Arbitrage and the Behaviour of Stock Index Futures Prices. Review of Financial Studies 1, 137-158.

[27] Markowitz H.M. (1952). The utility of wealth. Journal of Political Economy 60, 151-156.

[28] Modest D.M., Sundaresan M. (1983). The Relationship between Spot and Futures Prices in Stock Index Futures Markets, Some Preliminary Evidence. Journal of Futures Markets 3, 1541.

[29] Muller, A., Scarsini, M., Tsetlin, I., Winkler, R.W. (2015). Between First and Second Stochastic Dominance. INSEAD Working Paper Series 2015/55/DSC.

[30] Osuna, E.E. (2013). Tail-restricted stochastic dominance. IMA Journal of Management Mathematics 24, 21-44. 
[31] Popescu, C.C., Fulga, C. (2011). Possibilistic Optimization with Applications to Portfolio Selection. Proceedings of the Romanian Academy Series A: Mathematics, Physics, Technical Sciences, Information Science, 2, 88-94.

[32] Rieger M.O. (2011). Co-monotonicity of optimal investments and the design of structured financial products, Finance and Stochastics, 15: 27-55.

[33] Rieger M.O. (2012). Why Do Investors Buy Bad Financial Products? Probability Misestimation and Preferences in Financial Investment Decision. Journal of Behavioral Finance, 13, 108-118.

[34] Ritter J.R. (2003). Behavioral finance. Pacific-Basin Finance Journal 11, 429-437.

[35] Rothschild M., Stiglitz J.E. (1970). Increasing risk: I. definition, Journal of Economic Theory $2,225-243$.

[36] Scarsini, M. (1986). Comparison of random cash flows. IMA Journal of Management Mathematics 1, 25-32.

[37] Sriboonchitta S., Wong W.K., Dhompongsa S., Nguyen H.T. (2010). Stochastic Dominance and Applications to Finance, Risk and Economics. Chapman \& Hall/CRC, Boca Raton, USA.

[38] Starmer, C., Sugden, R. (1989). Probability and Juxtaposition Effects: an Experimental Investigation of the Common Ratio Effect. Journal of Risk and Uncertainty 2, 159-178.

[39] Yadav P.K., Pope P.F. (1994). Stock Index Futures Mispricing, Profit Opportunities or Risk Premia?. Journal of Banking and Finance 18, 921-53.

[40] Wallmeier M. (2011). Beyond payoff diagrams: how to present risk and return characteristics of structured products. Financial Markets and Portfolio Management, 25:313-338.

[41] Wang, M., Fishbeck, P.S. (2004). Incorporating Framing into Prospect Theory Modeling: A Mixture-Model Approach. Journal of Risk and Uncertainty 29, 181-197.

[42] Wilmott P. (2000). Paul Wilmott on Quantitative Finance. John Wiley \& Sons Ltd.

[43] Wu, G. (1994). An Empirical Test of Ordinal Independence. Journal of Risk and Uncertainty 9, 39-60. 